\title{
Article \\ Risk-Based Virtual Power Plant Implementation Strategy for Smart Energy Communities
}

\author{
Eunsung Oh
}

Citation: Oh, E. Risk-Based Virtual Power Plant Implementation Strategy for Smart Energy Communities. Appl. Sci. 2021, 11, 8248. https://doi.org/ 10.3390/app11178248

Academic Editor: Alberto Benato

Received: 30 July 2021

Accepted: 3 September 2021

Published: 6 September 2021

Publisher's Note: MDPI stays neutral with regard to jurisdictional claims in published maps and institutional affiliations.

Copyright: (C) 2021 by the author. Licensee MDPI, Basel, Switzerland. This article is an open access article distributed under the terms and conditions of the Creative Commons Attribution (CC BY) license (https:// creativecommons.org/licenses/by/ $4.0 /)$.
Department of Electrical and Electronic Engineering, Hanseo University, Seosan-si 31962, Chungcheongnam-do, Korea; esoh@hanseo.ac.kr

\begin{abstract}
This paper focuses on a virtual power plant (VPP) implementation strategy for smart local energy communities (SECs) with energy service providers. It is difficult to balance energy in the implementation stage due to uncertainties in demand and resources. Therefore, VPP implementation was modeled using the risk factor of energy balance. Using this risk factor, it was shown that the temporal correlation between demand and resources was the dominant factor involved in VPP implementation. Based on this, two risk-based VPP implementation strategies are proposed: an optimization-based strategy and a simple strategy that is solved in an iterative way. To minimize VPP implementation costs, the proposed strategies select the resources that have high correlation coefficients with demand and low correlation coefficients with other resources. Experimental results using real data sets show that the proposed strategies based on the risk factor are effective means of VPP implementation for commercial and residential SECs. The results imply that VPPs for commercial SECs are possible when PV is used as the main resource and is supplemented by wind, and it is effective to configure VPPs for residential SECs using wind according to the correlation between demand and resources.
\end{abstract}

Keywords: energy management system; implementation; operation; renewable; smart energy community; smart grid; virtual power plant

\section{Introduction}

The power sector, responsible for the generation of electricity and heat, produces the most greenhouse gas emissions at about $30 \%$ of total global greenhouse gas emissions [1] and $40 \%$ of energy-related $\mathrm{CO}_{2}$ emissions [2]. To reduce this, the use of renewables as generation resources is rapidly increasing. Renewables generated $28 \%$ of global power in 2018 with major contributions by wind power, solar power and hydropower [3]. It is expected that renewable power capacity will expand by more than $50 \%$ between 2019 and 2024 , and renewables will meet $80 \%$ of the growth in global electricity demand until $2030[4]$.

However, an increase in renewables with intermittent and uncontrollable characteristics makes grid operation difficult. It is reported that the changing resource mix of renewables is the highest threat to grid reliability [5]. The uncertainty of renewables not only reduces their utilization but also acts as a burden on system operation through issues including increases in reserved margins and operational complexity.

A virtual power plant (VPP) virtually interconnects various resources through a central control unit, but resources remain physically independent in their ownership and operation [6]. VPPs help establish stable power systems with integration of various types of resources [7]. Types of resources can include roof-top photovoltaic (PV), solar farms, wind farms, combined heat and power units, storage systems and flexible power consumers. The objective of VPPs is to relieve the load on the grid by smartly distributing the power generated by individual units.

Many studies have been researched on VPPs, applying technical optimization-based [8-10], meta-heuristic [11-14], game-theoretic [15-17], and deep-learning-based [18,19] approaches 
as well as systematically considering irrigation systems [9], buildings [20], electric vehicles [21] and power-to-gas [22]. Yi et al. considered VPP scheduling including day-ahead bidding and real-time operation to maximize economic benefits [8]. It was formulated as a mixed-integer, nonlinear programming problem and was solved effectively through a clustering-based load aggregation method and a disaggregation method for deferrable loads, satisfying consumers' diversified requirements. Naval et al. proposed an optimal technical-economic dispatch model of a VPP that combined large- and small-scale distributed renewable generation [9]. The problem was solved using mixed-integer linear programming. Elgamal et al. presented a comparative analysis of various scales of virtual power plant models [10]. They formulated a VPP operation problem considering dayahead and balancing markets and solved it by applying mixed-integer linear programming. Hadayeghparast et al. formulated multi-objective VPP scheduling to maximize profits and minimize emissions as a non-linear and non-convex problem [11]. The problem was heuristically solved by applying a particle swarm optimization (PSO) algorithm. Zhang et al. formulated a bi-objective VPP dispatch problem to optimize economic costs and power quality [12]. A long short-term memory method was applied to increasing the accuracy of wind prediction, and a multi-objective PSO algorithm was utilized as the solving algorithm. Hannan et al. introduced an optimal schedule controller to manage renewable energy resources in VPPs [13]. It was also solved using binary PSO. Pal et al. suggested the optimal scheduling of generation in VPPs for a day-ahead market framework using the beetle antenna search algorithm under various scenarios [14]. Yin et al. proposed a two-stage, robust Stackelberg game considering the uncertainties of renewable energy output and market prices for optimal scheduling in a VPP [15]. Wang et al. conducted a feasibility study of urban virtual power plants with the goal of energy self-sufficiency and applied Shapley value-based cooperative game theory, aiming to benefit both the plant and demand sides [16]. Jafari and Foroud presented a VPP coalition-forming model based on auctions considering the competition between VPPs in auctions for attracting distributed energy resources [17]. Sadeghi et al. proposed an optimal bidding strategy for a VPP for participating in electricity markets [18]. A deep-learning-based approach was employed to handle the uncertainties. Lin et al. suggested a deep reinforcement learning algorithm as the optimal online economic dispatch strategy for VPPs and designed an edge computing framework to handle the stochastic and large-state space characteristics of VPPs [19]. Moreover, comprehensive reviews of VPP research were conducted in $[6,23,24]$. Zhang et al. reviewed the research on VPPs by dividing it into internal control and external market bidding strategies [6]. Internal control involves resource dispatch, and it is divided into centralized, comprehensive and distributed control by adopting a control agent. The external market bidding strategy is related to the economic effects of the participation of VPPs in electricity markets, and it is mainly formulated as a mixed-integer linear problem solved by optimization or heuristic methods. Naval and Yusta also divided the objectives of VPP research into energy management to optimize scheduling resources and bidding strategies [23]. The review concludes that most of the research has been focused on the development of VPP models to achieve optimal control and coordination among their components and thus maximize operating profits. Rouzbahani et al. presented a detailed review of extracted documents regarding optimal scheduling techniques, technical and economic limitations and different kinds of uncertainties [24]. This paper focuses on the operation scheduling problem, which plays a significant role in the VPP concept.

However, most of the research focused on VPP operation involves how to dispatch resources to maximize benefits or minimize costs. VPPs operate using aggregated resources. This means that in VPP implementation, the resources gathered determine the operation room of the VPP. For example, if a VPP consists of only PV resources, VPP operation cannot be performed at night. A few works touch upon VPP implementation $[25,26]$. Masoud et al. researched a distributed generation sizing problem considering the price of electricity sales for increasing grid reliability and suggested a heuristic solution applying PSO [25]. Duan et al. proposed a VPP construction model based on decision area division [26]. They 
introduced a fusion space distance that accounted for geographical information and load density. However, these studies do not sufficiently consider the characteristics of resources, especially temporal characteristics. Renewables, the main resources constituting VPPs, repeat over time. Therefore, it is necessary to consider temporal characteristics when implementing VPPs.

This paper focuses on VPP implementation for smart energy communities (SECs). SECs are local energy communities $[27,28]$. SECs can be divided into residential SECs centered on households and commercial SECs, consisting of office buildings. SECs have their own energy service providers, so they can choose to supply power using VPPs and the utility grid [29]. A VPP serves the demand of an SEC. However, it is difficult to balance energy in its implementation stage due to uncertainties of demand and resources. Therefore, at the VPP implementation stage, the energy imbalance between the demand and the resources is modeled as the risk factor. Through the risk factor, it is shown that the temporal correlation between demand and resources is the dominant factor in VPP implementation. Based on that, two risk-based VPP implementation strategies are proposed: an optimization-based strategy and a simple strategy. The optimization-based strategy is designed to minimize VPP implementation costs, and the risk factor is relaxed using the relaxation multiplier. It can be optimally solved using linear programming. The simple strategy is designed to obtain an iterative approach to reduce computation complexity. The VPP selects resources in a one-by-one manner according to their correlation with demand. Experimental results using real data sets show that the proposed strategies based on the risk factor are effective means of VPP implementation for commercial and residential SECs.

The rest of this paper is organized as follows: In Section 2, the VPP for the SEC model is described, and in Section 3, the design method of the proposed risk-based VPP implementation strategy is discussed. In Section 4, measurement studies applied to the proposed strategy are presented, and in Section 5, lessons and future research directions are discussed. In Section 6, the conclusions of the paper are presented.

\section{Virtual Power Plants for Smart Energy Communities}

A VPP for an SEC consists of three parts: the VPP, the SEC and the utility grid, as shown in Figure 1. A VPP is configured and operated by a VPP service provider (SP). The VPP's SP recruits generation resources for the VPP's service. Generation resources for the VPP include individual power generators such as PVs, wind turbines and electric vehicles. Aggregated resources are utilized to supply power to VPP service participants such as SECs. An SEC consists of community members and the smart energy service provider (SESP) [29]. The community members are residential or commercial units. The SESP is a demand aggregator similar to the VPP SP. The SESP manages the energy transactions between members and energy producers such as between the VPP SP and the utility grid. Through the contract between the VPP SP and the SESP, the community members participate in power service from the VPP. The power generated by the resources in the VPP is transmitted to the SEC members through the utility grid. For energy balance, the SEC is also connected to the utility grid.

The main problem in a VPP for an SEC is how to effectively serve the power consumption of the SEC using the resources in the VPP. The VPP serves according to the capacity limit of resources. The capacity limit is determined according to what kind and size of resource the VPP has implemented. If the capacity is insufficient, it will not be able to provide adequate service, and if there is too much capacity, the burden of operating cost is increased. Therefore, VPP implementation is a prerequisite for effective VPP operation. 


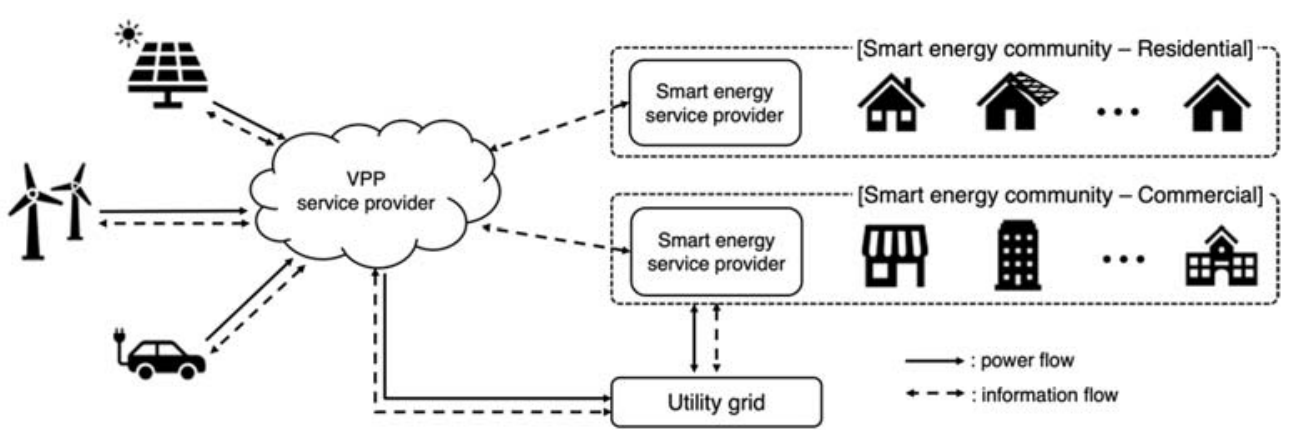

Figure 1. Constitution of a virtual power plant for smart energy communities.

\section{Risk-Based VPP Implementation Strategy}

\subsection{Baseline Problem}

In this subsection, a baseline problem in VPP implementation is described. In the baseline problem, it is assumed that the power consumption of an SEC and the power generation of resources are known at the VPP implementation stage. The solution of this problem can be used to verify the feasibility of the proposed VPP implementation strategy.

Let $c_{i}$ and $p_{i}$ be the generation capacity and the unit implementation cost of resource $i$, respectively. The VPP implementation cost is measured as

$$
O(\mathbf{c})=\sum_{i \in \mathcal{I}} p_{i} c_{i}
$$

where $\mathcal{I}$ is a set of resources $\mathcal{I}=\{1, \cdots, i, \cdots, I\}$, and $\mathbf{c}=\left\{c_{1}, \cdots, c_{i}, \cdots, c_{I}\right\}$.

When the VPP service participant of the SEC requires power consumption $d_{t}$, the VPP should support demand using the power generation of resources $i, g_{t}^{i}$, as follows:

$$
d_{t}=\sum_{i \in \mathcal{I}} g_{t}^{i}, \quad \forall t \in \mathcal{T}
$$

where $\mathcal{T}$ is the VPP service time as $\mathcal{T}=\{1, \cdots, t, \cdots, T\}$.

The generated power from resource $i$ is limited within the generation capacity purchased by the VPP:

$$
g_{t}^{i} \leq c_{i} \quad \forall t \in \mathcal{T}, i \in \mathcal{I} .
$$

Therefore, the VPP implementation problem regarding the capacity of resources to purchase to serve the requirements of demand is theoretically formulated as follows:

$$
\begin{array}{rr}
\text { P0 : } \quad \min _{\mathbf{c}} & O(\mathbf{c})=\sum_{i \in \mathcal{I}} p_{i} c_{i}, \\
\text { subject to } & d_{t}=\sum_{i \in \mathcal{I}} g_{t}^{i}, \quad \forall t \in \mathcal{T}, \\
& g_{t}^{i} \leq c_{i} \forall t \in \mathcal{T}, i \in \mathcal{I} .
\end{array}
$$

Problem P0 in (4) appears to be a simple linear problem. This is because it is assumed that the power consumption and generation are known at the VPP implementation stage. However, the generated power $g_{t}^{i}$ and the demand $d_{t}$ are not deterministic values. To optimally solve this problem, information including future-generated and demand values during service time $\mathcal{T}$ is required at the VPP implementation stage, which is not possible. This is the difficulty in applying the solution of the VPP implementation problem to a practical system. In this paper, to overcome this difficulty, problem $\mathbf{P 0}$ is relaxed based on the risk factor, and a risk-based VPP implementation strategy is proposed.

\subsection{Risk-Based VPP Implementation Strategy}

As mentioned above, the generated power from renewables and the demand of the SEC are uncertain. Therefore, it is impossible to meet the energy balance of the first 
constraint in problem P0 at the VPP implementation stage. To design the VPP implementation strategy, the energy balance constraint is relaxed, becoming a risk function that can be measured using the expected demand and generation. Based on these factors, an optimization-based VPP implementation strategy is proposed. Moreover, to reduce computational complexity, a simple VPP implementation strategy is suggested to obtain an iterative way of solving the problem.

\subsubsection{Optimization-Based VPP Implementation Strategy}

In this study, the energy balance constraint is relaxed as risk function

$$
F\left(\mathbf{c}, \hat{\mathrm{d}}, \hat{\mathrm{g}}^{i}\right)=\frac{1}{T} \sqrt{\left(\hat{\mathrm{d}}-\sum_{i \in \mathcal{I}} \lambda_{i} \hat{\mathrm{g}}^{i}\right)^{2}},
$$

where $\hat{\mathrm{d}}$ and $\hat{\mathrm{g}}^{i}$ are the expected demand and generation of resource $i$ in the form of column vectors $\hat{\mathrm{d}}=\left\{\hat{d}_{1}, \ldots, \hat{d}_{t}, \ldots, \hat{d}_{T}\right\}^{T}$ and $\hat{\mathrm{g}}^{i}=\left\{{\hat{g_{1}}}^{i}, \ldots,{\hat{g_{t}}}^{i}, \ldots, \hat{g}_{T}{ }^{i}\right\}^{T}$. The expectation values can be measured using the historic data for demand and generation. $\lambda_{i}$ is the capacity factor according to the second constraint in problem P0. The generation supplied from resource $i$ is proportional to the capacity purchased by the VPP. Therefore, the second constraint in problem $\mathbf{P 0}$ is modified as the capacity factor related to the maximum capacity of resource $i, \lambda_{i}=c_{i} / c_{i}^{\max }$.

The risk function of the energy balance can be rewritten as a vector form:

$$
\begin{aligned}
F\left(\mathbf{c}, \hat{\mathrm{d}}, \hat{\mathrm{g}}^{i}\right)= & \frac{1}{T} \sqrt{\left(\hat{\mathrm{d}}^{T} \hat{\mathrm{d}}-\sum_{i \in \mathcal{I}} \lambda_{i}^{2} \hat{\mathrm{g}}^{i T} \hat{\mathrm{g}}^{i}-2 \sum_{i \in \mathcal{I}} \lambda_{i} \hat{\mathrm{d}} \hat{\mathrm{g}}^{i}+2 \sum_{i \in \mathcal{I}} \sum_{j \in \mathcal{J}} \lambda_{i} \lambda_{j} \hat{\mathrm{g}}^{i T} \hat{\mathrm{g}}^{j}\right),} \\
= & \frac{1}{T} \sqrt{\hat{\mathrm{d}}^{T} \hat{\mathrm{d}}+\sum_{i \in \mathcal{I}} \lambda_{i}\left(-\lambda_{i} \hat{\mathrm{g}}^{i T} \hat{\mathrm{g}}^{i}-2 \hat{\mathrm{d}} \hat{\mathrm{g}}^{i}+2 \sum_{j \in \mathcal{J}} \lambda_{j} \hat{\mathrm{g}}^{i T} \hat{\mathrm{g}}^{j}\right) .}
\end{aligned}
$$

In the risk function, the first term, $\hat{d}^{T} \hat{d}$, is only related to the demand of the SEC. Therefore, the risk function of resource $i$ when implementing a VPP can be defined as follows:

$$
f_{i}\left(\mathbf{c}, \hat{\mathrm{d}}, \hat{\mathrm{g}}^{i}\right)=2 \sum_{j \in \mathcal{J}} \lambda_{j} \hat{\mathrm{g}}^{i T} \hat{\mathrm{g}}^{j}-\lambda_{i} \hat{\mathrm{g}}^{i T} \hat{\mathrm{g}}^{i}-2 \hat{\mathrm{d}} \hat{\mathrm{g}}^{i}
$$

The risk function of resource $i$ in (7) consists of two parts: (1) the temporal correlation between the expected generation of resource $i$ and the expected generation of other resources expressed as the first and second terms of $\sum_{j \in \mathcal{J}} \hat{\mathrm{g}}^{i T} \hat{\mathrm{g}}^{j}-\hat{\mathrm{g}}^{i T} \hat{\mathrm{g}}^{i}$ and (2) the temporal correlation between the expected generation of resource $i$ and the expected demand as the third term of $\hat{\mathrm{d}} \hat{\mathrm{g}}^{i}$. This provides the following guide for VPP implementation to reduce the energy balance risk:

1. The generated resources in the VPP should have low temporal correlation with each other;

2. The generation of resources in the VPP should have a high temporal correlation with the served participant's demand.

Using the risk function, problem $\mathbf{P 0}$ is relaxed as an unconstrained problem:

$$
\text { P1 : } \min _{\mathbf{c}} \widetilde{O}\left(\mathbf{c}, \hat{\mathrm{d}}, \hat{\mathrm{g}}^{i}\right)=O(\mathbf{c})+\gamma F\left(\mathbf{c}, \hat{\mathrm{d}}, \hat{\mathrm{g}}^{i}\right),
$$

where $\gamma$ is the relaxation multiplier, which imposes the priority of the risk. Problem P1 is quadratic and differentiable. Therefore, it can be optimally solved using linear programming [30]. 


\subsubsection{Simple VPP Implementation Strategy}

Using the guide presented in the risk function design, a simple VPP implementation strategy can be suggested to solve problem P1. As discussed in the risk function design, the temporal correlation between generation in the VPP and serving demand is the dominant factor when determining the risk. The risk factor of each resource expresses the cost of the risk. Therefore, as the first step of the risk-based VPP implementation strategy, a resource is selected to minimize the risk factor. The VPP SP purchases a fixed capacity of the selected resource to implement the VPP in the second step. In the third step, due to the addition of capacity, the serving demand is updated, excluding the amount served. Using the risk factor that considers the updated serving demand and generation in the VPP, resources are repeatedly selected until the objective function converges. A flow chart of the risk-based VPP implementation strategy is presented in Figure 2.

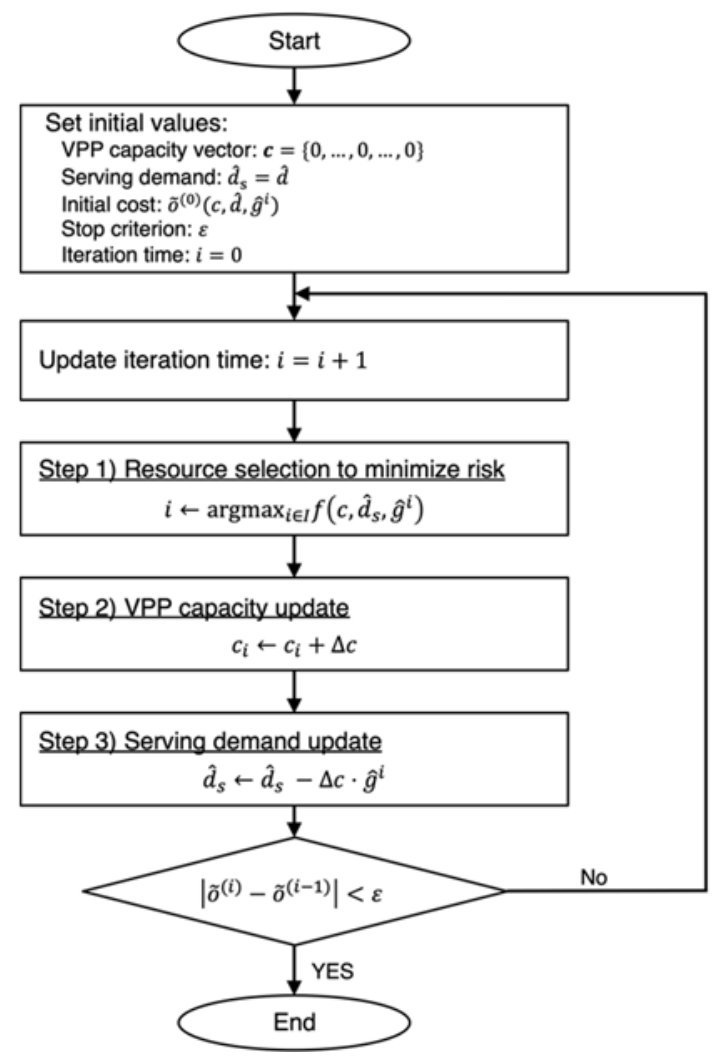

Figure 2. Flow chart of the simple risk-based VPP implementation strategy.

\section{Results}

In this section, to verify the effectiveness of the proposed strategy, the performance of the VPP implementation is measured, and the effects of the characteristics of the system parameters are discussed.

To perform simulations, $10 \mathrm{PV}$ and 10 wind generations were considered as resources that could compose a VPP. The actual generation data used for the resources were recorded at the Lawrence Berkeley National Laboratory in the US for PV [31] and the National Renewable Energy Laboratory in the US for wind [32]. The unit implementation costs of the resources were assumed to be $\$ 5.83 / \mathrm{kW} /$ month for community-sized PV and $\$ 5.21 / \mathrm{kW} /$ month for onshore wind, considering a service period of 20 years [33].

The demand of the SEC was modeled using commercial and residential hourly load profiles in the US [34]. The average daily demands of commercial SECs and residential SECs are about $1500 \mathrm{kWh}$ and $600 \mathrm{kWh}$, respectively. Figure 3 shows the typical demand of a commercial SEC and a residential SEC for a week. As shown in the figure, the commercial SEC requires high demand on weekdays and low demand on weekends. On the other 
hand, the demand of the residential SEC shows a similar repetition pattern over a week. In addition, in the case of the commercial SEC, peak demand appears in the daytime, and in the residential SEC, it appears in the evening. The demand characteristics affect the implementation of the VPP.

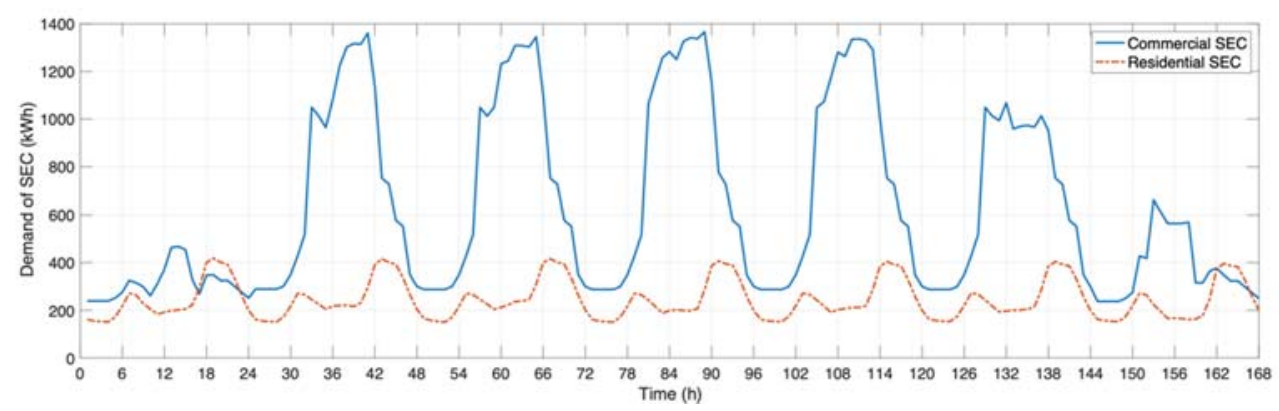

Figure 3. Typical demand of a commercial SEC (blue line) and a residential SEC (dashed red line) for a week.

Figure 4 shows an example of VPP implementation for a commercial SEC. The black line, the dashed red line and the blue dash-dot line red represent the required demand of a commercial SEC, VPP-served PV generation and VPP-served PV and wind generation, respectively. As shown in the figure, the demand is mainly served by PV generation, and it is supplemented by wind generation. This is because the demand of a commercial SEC and PV generation have a high temporal correlation. However, on weekends, more generation than demand is supplied, and energy mismatching occurs at other times due to uncertainty of resources. This energy mismatching is resolved through the utility grid.

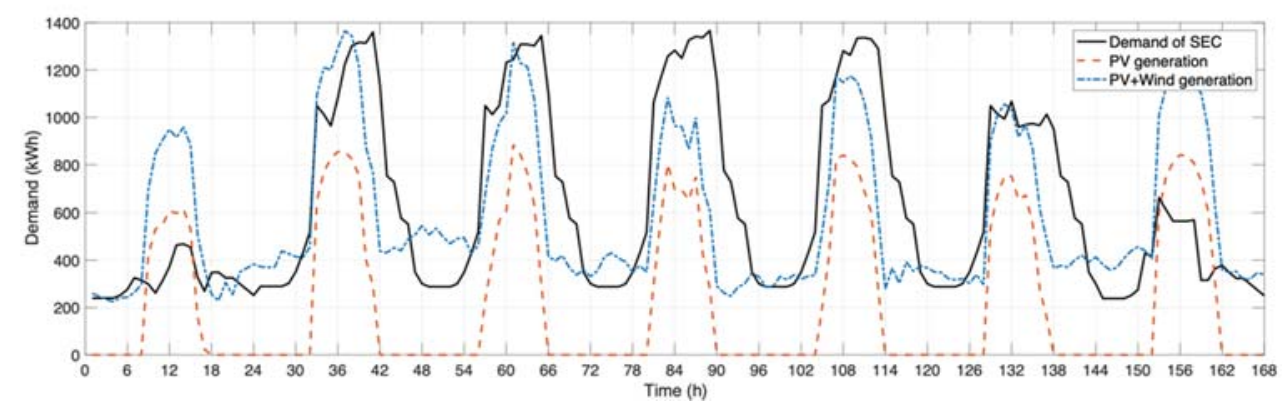

Figure 4. An example of VPP implementation for a commercial SEC.

\subsection{Effectiveness of Risk-Based VPP Implementation}

The VPP implementation results are presented in Table 1 for the commercial SEC and in Table 2 for the residential SEC. P0 and P1 show the solutions to the problems in (4) and in (8), respectively. P2 expresses the results of the simple VPP implementation strategy presented in Figure 2.

In Tables 1 and 2, the P0 VPP implementation result has the largest resource capacity. In $\mathbf{P 1}$ and P2, more capacity is constructed as the relaxation multiplier $\gamma$ increases. With the highest relaxation multiplier of $\gamma=1$, the implementation results of $\mathbf{P 0}$ and $\mathbf{P} \mathbf{1}$ suggest similar solutions. Moreover, the results of $\mathbf{P 2}$, as solved by the simple VPP implementation strategy, configure the VPP by selecting the resource used in $\mathbf{P 0}$ and P1. 
Table 1. VPP implementation results for a commercial SEC.

\begin{tabular}{cccccccc}
\hline $\begin{array}{c}\text { Method } \\
\text { Resource }\end{array}$ & $\mathbf{P 0}$ & $\begin{array}{c}\mathbf{P 1} \\
(\gamma=0.1)\end{array}$ & $\begin{array}{c}\mathbf{P 1} \\
(\boldsymbol{\gamma}=0.5)\end{array}$ & $\begin{array}{c}\mathbf{P 1} \\
(\boldsymbol{\gamma}=1)\end{array}$ & $\begin{array}{c}\mathbf{P 2} \\
(\gamma=0.1)\end{array}$ & $\begin{array}{c}\mathbf{P 2} \\
(\gamma=0.5)\end{array}$ & $\begin{array}{c}\mathbf{P 2} \\
(\gamma=1)\end{array}$ \\
\hline PV1 & 0 & 0 & 0 & 0 & 0 & 0 & 0 \\
PV2 & 740 & 757 & 852 & 807 & 410 & 515 & 535 \\
PV3 & 0 & 0 & 0 & 0 & 0 & 0 & 0 \\
PV4 & 240 & 0 & 128 & 196 & 45 & 170 & 190 \\
PV5 & 0 & 0 & 0 & 0 & 0 & 0 & 0 \\
PV6 & 0 & 0 & 0 & 0 & 0 & 0 & 0 \\
PV7 & 0 & 0 & 0 & 0 & 0 & 0 & 0 \\
PV8 & 0 & 0 & 0 & 24 & 0 & 0 & 0 \\
PV9 & 0 & 0 & 0 & 0 & 0 & 0 & 0 \\
PV10 & 247 & 0 & 0 & 0 & 0 & 0 & 0 \\
Wind1 & 224 & 154 & 207 & 212 & 0 & 0 & 0 \\
Wind2 & 28 & 241 & 45 & 45 & 565 & 565 & 565 \\
Wind3 & 0 & 0 & 0 & 0 & 0 & 0 & 0 \\
Wind4 & 0 & 0 & 0 & 0 & 0 & 0 & 0 \\
Wind5 & 0 & 0 & 0 & 0 & 0 & 0 & 0 \\
Wind6 & 395 & 281 & 363 & 385 & 35 & 180 & 205 \\
Wind7 & 148 & 0 & 155 & 146 & 0 & 0 & 0 \\
Wind8 & 0 & 0 & 0 & 0 & 0 & 0 & 0 \\
Wind9 & 0 & 0 & 0 & 0 & 0 & 0 & 0 \\
Wind10 & 0 & 0 & 0 & 0 & 0 & 0 & 0 \\
\hline PV total & 1227 & 757 & 980 & 1027 & 455 & 685 & 725 \\
Wind & 795 & 676 & 770 & 788 & 600 & 745 & 770 \\
total & & & & & & & \\
\hline
\end{tabular}

Table 2. VPP implementation results for a residential SEC.

\begin{tabular}{cccccccc}
\hline $\begin{array}{c}\text { Method } \\
\text { Resource }\end{array}$ & $\mathbf{P 0}$ & $\begin{array}{c}\mathbf{P 1} \\
(\gamma=0.1)\end{array}$ & $\begin{array}{c}\mathbf{P 1} \\
(\gamma=0.5)\end{array}$ & $\begin{array}{c}\mathbf{P 1} \\
(\gamma=1)\end{array}$ & $\begin{array}{c}\mathbf{P 2} \\
(\gamma=0.1)\end{array}$ & $\begin{array}{c}\mathbf{P 2} \\
(\gamma=0.5)\end{array}$ & $\begin{array}{c}\mathbf{P 2} \\
(\gamma=1)\end{array}$ \\
\hline PV1 & 0 & 0 & 0 & 0 & 0 & 0 & 0 \\
PV2 & 0 & 0 & 0 & 0 & 0 & 0 & 0 \\
PV3 & 0 & 0 & 0 & 0 & 0 & 0 & 0 \\
PV4 & 0 & 0 & 0 & 0 & 0 & 0 & 0 \\
PV5 & 0 & 0 & 0 & 0 & 0 & 0 & 0 \\
PV6 & 0 & 0 & 0 & 0 & 0 & 0 & 0 \\
PV7 & 0 & 0 & 0 & 0 & 0 & 0 & 0 \\
PV8 & 0 & 0 & 0 & 0 & 0 & 0 & 0 \\
PV9 & 0 & 0 & 0 & 0 & 0 & 0 & 0 \\
PV10 & 0 & 0 & 0 & 0 & 0 & 0 & 0 \\
Wind1 & 205 & 161 & 195 & 204 & 75 & 80 & 80 \\
Wind2 & 41 & 145 & 70 & 51 & 250 & 250 & 250 \\
Wind3 & 0 & 0 & 0 & 0 & 0 & 0 & 0 \\
Wind4 & 0 & 24 & 0 & 0 & 0 & 0 & 0 \\
Wind5 & 0 & 0 & 0 & 0 & 0 & 0 & 0 \\
Wind6 & 117 & 38 & 103 & 108 & 0 & 20 & 25 \\
Wind7 & 89 & 2 & 65 & 81 & 0 & 0 & 0 \\
Wind8 & 0 & 0 & 0 & 0 & 0 & 0 & 0 \\
Wind9 & 0 & 0 & 0 & 0 & 0 & 0 & 0 \\
Wind10 & 0 & 0 & 0 & 0 & 0 & 0 & 0 \\
\hline PV total & 0 & 0 & 0 & 0 & 0 & 0 & 0 \\
Wind & 452 & 370 & 433 & 444 & 325 & 350 & 355 \\
total & & & & & & & 0 \\
\hline
\end{tabular}

\subsection{Effects of Demand}

The resources for implementing a VPP change according to the characteristics of demand. In the VPP implementation servicing the demand of a commercial SEC, PV 
resources were largely utilized, and wind resources were compensated, as shown in Table 1. To check Pearson's linear correlation coefficient (PLCC) [35], the demand of the commercial SEC and the PV resources had a high correlation coefficient, as shown in Table 3. Among them, the PV2 and PV4 resources with the highest and the second highest correlation coefficients were used for the VPP implementation serving the demand of the commercial SEC. For wind resources, Wind2 and Wind7, negatively correlated to the demand of the commercial SEC, were used in addition to Wind1 and Wind6, which had positive correlations with the demand of the commercial SEC. In particular, in the results for P2, Wind2, which had low correlation with the existing constituent resources, was used more compared to the results for other methods.

Table 3. Pearson's linear correlation coefficient between demand and resources.

\begin{tabular}{ccc}
\hline Resource & Demand of Commercial SEC & Demand of Residential SEC \\
\hline PV1 & 0.28 & -0.03 \\
PV2 & 0.66 & -0.03 \\
PV3 & 0.60 & -0.33 \\
PV4 & 0.62 & -0.30 \\
PV5 & 0.60 & -0.32 \\
PV6 & 0.60 & -0.32 \\
PV7 & 0.59 & -0.32 \\
PV8 & 0.39 & -0.27 \\
PV9 & 0.43 & -0.26 \\
PV10 & 0.39 & -0.28 \\
Wind1 & 0.03 & 0.16 \\
Wind2 & -0.16 & -0.13 \\
Wind3 & -0.42 & -0.14 \\
Wind4 & -0.08 & 0.03 \\
Wind5 & -0.07 & -0.03 \\
Wind6 & 0.13 & -0.02 \\
Wind7 & -0.34 & -0.24 \\
Wind8 & -0.35 & -0.16 \\
Wind9 & -0.24 & -0.13 \\
Wind10 & -0.48 & -0.18 \\
\hline
\end{tabular}

As shown in Table 3, the PCCC between the demand of the residential SEC and the PV resources was negative. Therefore, the VPP was implemented only using wind resources to serve the demand of the residential SEC, as shown in Table 2. Similar to the case of the commercial SEC, Wind1, which had the highest correlation with demand, was mainly used to serve the demand of the residential SEC.

\subsection{Cost Analysis}

Table 4 shows monthly costs according to VPP service. VPPs based on renewable resources cannot fully service demand due to the uncontrollable nature of renewables. Therefore, energy balancing is performed through transactions with the utility grid. It was assumed that the price of electricity purchased from the utility grid was $\$ 0.133 / \mathrm{kWh}$, and the selling price was half that of the purchased electricity. This is the average price of electricity traded in the US in 2021 [36].

In the results for $\mathbf{P 0}$, the cost to the utility grid was lowest. However, as a great deal of capacity was required to match the energy balance, the VPP cost increased. Therefore, the overall cost increased in the results of $\mathbf{P 0}$ compared to the results of the risk-based approaches P1 and P2. Moreover, in the results of P1, with a relaxation multiplier of $\gamma=0.5$, the total cost was minimized. 
Table 4. Monthly costs according to VPP service (\$/month).

\begin{tabular}{cccccccc}
\hline Cost & P0 & $\begin{array}{c}\text { P1 } \\
(\gamma=0.1)\end{array}$ & $\begin{array}{c}\text { P1 } \\
(\gamma=0.5)\end{array}$ & $\begin{array}{c}\text { P1 } \\
(\gamma=1)\end{array}$ & $\begin{array}{c}\text { P2 } \\
(\gamma=0.1)\end{array}$ & $\begin{array}{c}\text { P2 } \\
(\gamma=0.5)\end{array}$ & $\begin{array}{c}\text { P2 } \\
(\gamma=1)\end{array}$ \\
\hline \multicolumn{7}{c}{ Commercial SEC } \\
\hline VPP & 11,304 & 7934 & 9723 & 10,092 & 5779 & 7876 & 8240 \\
Grid & 15,736 & 18,984 & 16,621 & 16,257 & 23,143 & 19,105 & 18,624 \\
Total & 27,041 & 26,918 & 26,344 & 26,349 & 28,922 & 26,981 & 26,864 \\
Unit & 0.060 & 0.060 & 0.059 & 0.059 & 0.064 & 0.060 & 0.060 \\
price & & & & & & \\
\hline & & & & & & \\
\hline VPP & 2353 & 1930 & 2255 & 2311 & 1693 & 1823 & 1849 \\
Grid & 5225 & 5746 & 5311 & 5264 & 6126 & 5816 & 5769 \\
Total & 7578 & 7675 & 7566 & 7575 & 7818 & 7639 & 7618 \\
Unit & 0.043 & 0.043 & 0.043 & 0.043 & 0.044 & 0.043 & 0.043 \\
price & & & & & & \\
\hline
\end{tabular}

In all cases, as the VPP was served, the unit price was reduced as compared to the cost of purchasing power from the utility grid. Particularly, in the case of the residential SEC, about $70 \%$ of the cost was saved compared to about a $55 \%$ reduction in the commercial SEC case. Figure 5 shows the demand purchasing ratios from the VPPs and the utility grid. In the case of the residential SEC, approximately $80 \%$ of the VPP was utilized similarly in all results, as shown in Figure 5b. However, in the case of the commercial SEC, the utilization of the VPP varied according to risk priority, as in Figure 5a.
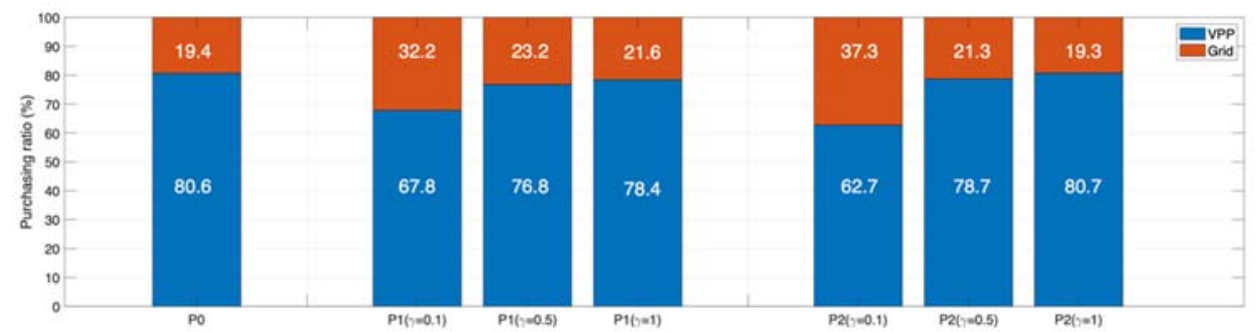

(a) Commercial SEC
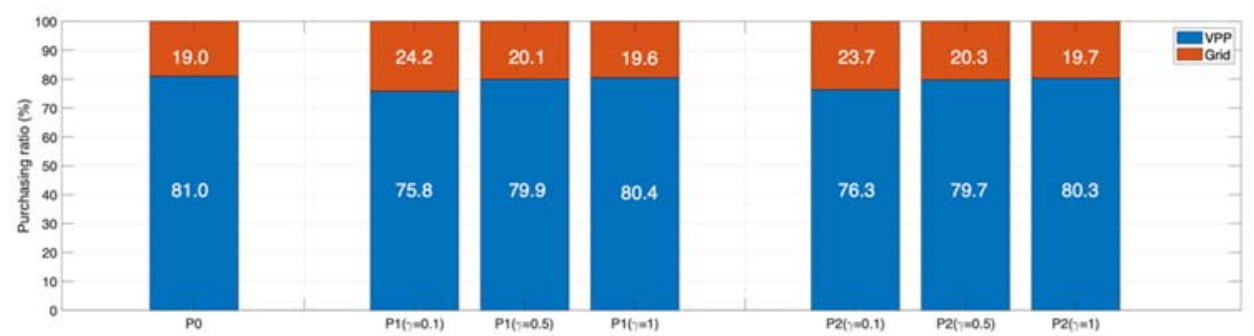

(b) Residential SEC

Figure 5. Demand purchasing ratios from the VPPs and the utility grid.

\section{Discussion}

\subsection{Effectiveness of the Risk-Based VPP Implementation}

Based on the VPP implementation results shown in Tables 1 and 2, the effectiveness of the risk-based VPP implementation can be discussed. From the values of P1 and $\mathbf{P 2}$, it can be seen that a higher capacity was obtained as the relaxation multiplier $\gamma$ increased. The energy balance was considered a risk factor in $\mathbf{P 1}$ and $\mathbf{P 2}$, and the relaxation multiplier imposed the priority of the risk. This indicates that when the priority of the risk was increased, more resources were required. It can also be confirmed that of the VPP implementation results, $\mathbf{P 0}$ had the largest resource capacity. This is because the 
energy balance was included as a constraint in the problem of P0. This shows that riskbased VPP implementation is effective. Moreover, the VPP was configured using the values of P0, P1 and P2 while selecting the same resources. This shows that a risk factor considering temporal correlation between the demand and resources acts as a dominant factor in VPP implementation. This further proves that the proposed, risk-based approach is well-designed.

\subsection{Effects of Demand}

To verify the PLCCs between the demand and the resources in Table 3, the resources with high correlation coefficients were selected for the VPP implementation, but some resources with negative correlations were also utilized. As an example, in the VPP implementation results for the commercial SEC in Table 1, PV2 and PV4 were selected as the resources for the VPP. This is because these resources had the highest and second-highest correlation coefficients with demand, as shown in Table 3. The high correlation means that the generation was high at the times of high demand. By utilizing resources with high correlations to demand, VPPs can provide stable services according to the needs of participants. However, Wind2, which has a negative correlation coefficient with demand, was also used for the VPP implementation for the commercial SEC. The negative correlation means that the generation of resources was counter to the requirements of the demand. It is inefficient to provide services by utilizing resources with negative correlations. However, in order to cover the residual demand that could not be served by resources with high correlations such as PV2 and PV4, a resource that was generated at a different time than the existing resources was required. This is why VPP implementation is affected by the correlation between demand and resources as well as the correlations among resources. In particular, the values of $\mathbf{P} \mathbf{2}$ are more significant when compared with other methods. This is because the risk factor, which is the basis of $\mathbf{P 2}$, is constructed based on the correlations of the resources as well as the correlation between the demand and resources. However, the results show that the correlation between the demand and a resource is important in VPP implementation. In particular, in the case of the commercial SEC, the PV-oriented VPP implementation was possible due to its high correlation with PV resources. In the case of the residential SEC, the wind resource-based VPP implementation was more suitable for PV resources.

\subsection{Cost Analysis}

In all cases, as the VPP was served, the unit price was reduced as compared to the cost of purchasing power from the utility grid, as shown in Table 4 . This is because the levelized costs of energy of the renewable resources was lower than the electricity cost of the utility grid. Particularly, the cost savings in the residential SEC were greater than those of the commercial SEC. This is because the demand of the residential SEC repeated without fluctuations for a week. The demand of the commercial SEC was high during the week, but the demand was low on the weekend, as shown in Figure 3. Accordingly, the energy produced could not be utilized over the weekend. In addition, in the case of the commercial SEC, the utilization of the VPP varied according to the risk priority shown in Figure 5. This is due to the characteristics of the demand described above. In the case of the commercial SEC, the utilization depended on the risk of mismatching that occurred over the weekend. This indicates that risk factor is an important indicator that determines cost.

\subsection{Brief Summary}

Through these results, lessons can be suggested for VPP implementation:

- Temporal correlation is an important factor in VPP implementation. There should be a high correlation coefficient between demand and resources and low correlation among resources;

- An efficient VPP implementation strategy can be suggested using risk factors based on correlation; 
- The demand of commercial SECs has a high correlation coefficient with PV. Accordingly, it is possible to implement VPPs in this form, using PV as the main resource, supplemented by wind;

- In the case of residential SECs, there is a negative correlation coefficient with PV. Therefore, it is effective to configure the VPP using wind resources.

This paper presents a risk-based VPP implementation strategy. By extending this, the following future research directions can be suggested:

- This paper focused on the VPP implementation problem. A co-optimization problem can be formulated considering both the risk of the VPP implementation and the energy balance during the VPP's operation;

- Flexible resources such as electric vehicles and energy storage can be considered as resources. Operations including energy storage charging and discharging should be considered flexible resources. Therefore, the problem with flexible resources can be formulated as a co-optimization problem.

\section{Conclusions}

This paper proposed VPP implementation strategies, considering their risk. It is difficult to balance energy in the implementation stage due to uncertainties in demand and resources. To reduce the difficulty, the VPP implementation was designed using a risk factor that reflected the energy imbalance risk between the demand and a resource. Using the risk factor, it was discovered that the temporal correlation between demand and resources was the dominant factor impacting VPP implementation. Particularly, it was shown that to reduce risk, a resource constituting part of the VPP should have a high correlation coefficient with the demand and a low correlation coefficient with other resources. Based on that, an optimization-based strategy was proposed. Moreover, a simple strategy that could be solved in an iterative way was suggested. Experimental results using the real data sets show that the proposed strategies based on the risk factor are effective means of VPP implementation for commercial and residential SECs. According to the characteristic of demand, PV and wind are suggested to be suitable resources for VPP implementation for commercial SECs and residential SECs, respectively.

VPP implementation is critical for VPP operation. This is because the resources constituting the VPP determine the operation room of the VPP. This means that a VP's operation method can influence the design of its implementation. Moreover, in terms of flexible resources, which increase the operating areas of VPPs, it is difficult to directly apply the correlation-based risk factor suggested in this study. Therefore, co-optimization that considers both the implementation and the operation of VPPs as well as the consideration of flexible resources for VPP implementation are future research directions.

Funding: This research was supported by the National Research Foundation of Korea with a grant funded by the Korean government (Ministry of Science and ICT) (No. 2020R1A2B5B01095044).

Institutional Review Board Statement: Not applicable.

Informed Consent Statement: Not applicable.

Data Availability Statement: Not applicable.

Conflicts of Interest: The author declares no conflict of interest. The funders had no role in the design of the study; in the collection, analyses, or interpretation of data; in the writing of the manuscript; or in the decision to publish the results.

\section{References}

1. Intergovernmental Panel on Climate. Climate Change 2014: Mitigation of Climate Change. Intergovernmental Panel on Climate Change (IPCC); Intergovernmental Panel on Climate: Geneva, Switzerland, 2014. [CrossRef]

2. International Energy Agency (IEA). Global Energy-Related $\mathrm{CO}_{2}$ Emissions by Sector. Available online: https: / www.iea.org/ data-and-statistics/charts/global-energy-related-co2-emissions-by-sector (accessed on 24 August 2021). 
3. U.S. Energy Information Administration. International Energy Outlook 2019 with Projections to 2050. 2019. Available online: https:/ / www.eia.gov/outlooks/ieo/pdf/ieo2019.pdf (accessed on 24 August 2021).

4. International Energy Agency (IEA). World Energy Outlook 2020. Available online: https://www.iea.org/reports/world-energyoutlook-2020 (accessed on 24 August 2021).

5. North American Electric Reliability Corporation (NERC). 2019 ERO Reliability Risk Priorities Report. 2019. Available online: https:/ / www.nerc.com/comm/RISC/Related\%20Files\%20DL/RISC\%20ERO\%20Priorities\%20Report_Board_Accpeted_ November_5_2019.pdf (accessed on 24 August 2021).

6. Zhang, G.; Jiang, C.; Wang, X. Comprehensive Review on Structure and Operation of Virtual Power Plant in Electrical System. IET Gener. Transm. Distrib. 2019, 13, 145-156. [CrossRef]

7. Fortune Business Insights. Virtual Power Plant: Global Market Analysis, Insights and Forecast, 2017-2028. Available online: https:/ / www.fortunebusinessinsights.com/industry-reports/virtual-power-plant-market-101669 (accessed on 24 August 2021).

8. Yi, Z.; Xu, Y.; Gu, W.; Wu, W. A Multi-Time-Scale Economic Scheduling Strategy for Virtual Power Plant Based on Deferrable Loads Aggregation and Disaggregation. IEEE Trans. Sustain. Energy 2019, 11, 1332-1346. [CrossRef]

9. Naval, N.; Sánchez, R.; Yusta, J.M. A Virtual Power Plant Optimal Dispatch Model with Large and Small-Scale Distributed Renewable Generation. Renew. Energy 2020, 151, 57-69. [CrossRef]

10. Elgamal, A.H.; Kocher-Oberlehner, G.; Robu, V.; Andoni, M. Optimization of a Multiple-Scale Renewable Energy-Based Virtual Power Plant in the UK. Appl. Energy 2019, 256, 113973. [CrossRef]

11. Hadayeghparast, S.; Farsangi, A.S.; Shayanfar, H. Day-Ahead Stochastic Multi-Objective Economic/Emission Operational Scheduling of a Large Scale Virtual Power Plant. Energy 2019, 172, 630-646. [CrossRef]

12. Zhang, J.; Xu, Z.; Xu, W.; Zhu, F.; Lyu, X.; Fu, M. Bi-Objective Dispatch of Multi-Energy Virtual Power Plant: Deep-Learning-Based Prediction and Particle Swarm Optimization. Appl. Sci. 2019, 9, 292. [CrossRef]

13. Hannan, M.; Abdolrasol, M.; Faisal, M.; Ker, P.J.; Begum, R.; Hussain, A. Binary Particle Swarm Optimization for Scheduling MG Integrated Virtual Power Plant toward Energy Saving. IEEE Access 2019, 7, 107937-107951. [CrossRef]

14. Pal, P.; Krishnamoorthy, P.A.; Rukmani, D.K.; Antony, S.J.; Ocheme, S.; Subramanian, U.; Elavarasan, R.M.; Das, N.; Hasanien, H.M. Optimal Dispatch Strategy of Virtual Power Plant for Day-Ahead Market Framework. Appl. Sci. 2021, 11, 3814. [CrossRef]

15. Yin, S.; Ai, Q.; Li, Z.; Zhang, Y.; Lu, T. Energy Management for Aggregate Prosumers in a Virtual Power Plant: A Robust Stackelberg Game Approach. Int. J. Electr. Power Energy Syst. 2020, 117, 105605. [CrossRef]

16. Wang, Y.; Gao, W.; Qian, F.; Li, Y. Evaluation of Economic Benefits of Virtual Power Plant Between Demand and Plant Sides Based on Cooperative Game Theory. Energy Convers. Manag. 2021, 238, 114180. [CrossRef]

17. Jafari, M.; Foroud, A.A. A Medium/Long-Term Auction-Based Coalition-Forming Model for a Virtual Power Plant Based on Stochastic Programming. Int. J. Electr. Power Energy Syst. 2020, 118, 105784. [CrossRef]

18. Sadeghi, S.; Jahangir, H.; Vatandoust, B.; Golkar, M.A.; Ahmadian, A.; Elkamel, A. Optimal Bidding Strategy of a Virtual Power Plant in Day-Ahead Energy and Frequency Regulation Markets: A Deep Learning-Based Approach. Int. J. Electr. Power Energy Syst. 2021, 127, 106646. [CrossRef]

19. Lin, L.; Guan, X.; Peng, Y.; Wang, N.; Maharjan, S.; Ohtsuki, T. Deep Reinforcement Learning for Economic Dispatch of Virtual Power Plant in Internet of Energy. IEEE Internet Things J. 2020, 7, 6288-6301. [CrossRef]

20. Royapoor, M.; Pazhoohesh, M.; Davison, P.J.; Patsios, C.; Walker, S. Building as a Virtual Power Plant, Magnitude and Persistence of Deferrable Loads and Human Comfort Implications. Energy Build. 2020, 213, 109794. [CrossRef]

21. Abbasi, M.H.; Taki, M.; Rajabi, A.; Li, L.; Zhang, J. Coordinated Operation of Electric Vehicle Charging and Wind Power Generation as a Virtual Power Plant: A Multi-Stage Risk Constrained Approach. Appl. Energy 2019, 239, 1294-1307. [CrossRef]

22. Ju, L.; Zhao, R.; Tan, Q.; Lu, Y.; Tan, Q.; Wang, W. A Multi-Objective Robust Scheduling Model and Solution Algorithm for a Novel Virtual Power Plant Connected with Power-to-Gas and Gas Storage Tank Considering Uncertainty and Demand Response. Appl. Energy 2019, 250, 1336-1355. [CrossRef]

23. Naval, N.; Yusta, J.M. Virtual Power Plant Models and Electricity Markets-A Review. Renew. Sustain. Energy Rev. 2021, 149, 111393. [CrossRef]

24. Rouzbahani, H.M.; Karimipour, H.; Lei, L. A Review on Virtual Power Plant for Energy Management. Sustain. Energy Technol. Assess. 2021, 47, 101370.

25. Maanavi, M.; Najafi, A.; Godina, R.; Mahmoudian, M.; MG Rodrigues, E. Energy Management of Virtual Power Plant Considering Distributed Generation Sizing and Pricing. Appl. Sci. 2019, 9, 2817. [CrossRef]

26. Duan, J.; Wang, X.; Gao, Y.; Yang, Y.; Yang, W.; Li, H.; Ehsan, A. Multi-Objective Virtual Power Plant Construction Model Based on Decision Area Division. Appl. Sci. 2018, 8, 1484. [CrossRef]

27. Ceglia, F.; Esposito, P.; Marrasso, E.; Sasso, M. From Smart Energy Community to Smart Energy Municipalities: Literature Review, Agendas and Pathways. J. Clean. Prod. 2020, 254, 120118. [CrossRef]

28. Bukovszki, V.; Magyari, á.; Braun, M.K.; Párdi, K.; Reith, A. Energy Modelling as a Trigger for Energy Communities: A Joint Socio-Technical Perspective. Energies 2020, 13, 2274. [CrossRef]

29. Oh, E.; Son, S.Y. Peer-to-Peer Energy Transaction Mechanisms Considering Fairness in Smart Energy Communities. IEEE Access 2020, 8, 216055-216068. [CrossRef]

30. Boyd, S.; Boyd, S.P.; Vandenberghe, L. Convex Optimization; Cambridge University Press: Cambridge, UK, 2004. 
31. Lawrence Berkeley National Laboratory (LBNL). Solar-to-Grid Public Data File for Utility-Scale (UPV) and Distributed Photovoltaics (DPV) Generation, Capacity Credit, and Value. Available online: https://data.openei.org/submissions/2881 (accessed on 24 August 2021).

32. National Renewable Energy Laboratory (NREL). Wind Resource Data, Tools, and Maps. Available online: https://www.nrel. gov/gis/wind.html (accessed on 24 August 2021).

33. LAZARD. Lazard's Levelized Cost of Energy Analysis, Version 14.0. 2020. Available online: https://www.lazard.com/media/45 1419/lazards-levelized-cost-of-energy-version-140.pdf (accessed on 24 August 2021).

34. Commercial and Residential Hourly Load Profiles for all TMY3 Locationus in the United States. Available online: https: / / data.openei.org/submissions/153 (accessed on 24 August 2021).

35. Lee Rodgers, J.; Nicewander, W.A. Thirteen Ways to Look at the Correlation Coefficient. Am. Stat. 1988, 42, 59-66. [CrossRef]

36. U.S. Energy Information Administration (EIA). Electric Power Monthly. Available online: https://www.eia.gov/electricity/ monthly/current_month/july2021.pdf (accessed on 24 August 2021). 